Rev. Biol. Trop., 47(3): 381-391, 1999

www.ucr.ac.cr www.ots.ac.cr www.ots.duke.edu

\title{
Las cianobacterias como indicadores de contaminación orgánica
}

\author{
Mariano Peinador \\ Instituto Costarricense de Acueductos y Alcantarillados. Apartado 175-2300 Curridabat Costa Rica. Fax: 225-9772
}

Recibido 2-IV-1998. Corregido 22-II-1999. Aceptado 5-V-1999.

\begin{abstract}
In two Costa Rican rivers used as receptors for domestic sewage, treated by primary stabilization ponds, were taken a total of 28 samplings located at the pond exit and at three different sites in each river: 100 $\mathrm{m}$ before the ponds discharge, at the discharge and $100 \mathrm{~m}$ after the discharge. These sampling were done for a five and a half years including dry and rainy seasons. In each sampling site, samples were collected of five different substrates: stones, submerge and semi submerge vegetation, tree trunks or sticks, water and artificial substrates. For each sample were used two types of artificial cultures, WC and BG110. A total of 55 cyanobacteria species isolations were obtained, belonging to a 26 genera, between these the most common were Phormidium with nine species, Microcystis with five species, Leptolyngbya and Pseudanabaena with four species each and Oscillatoria with three species. More cyanobacteria species were isolated in water substrate and less isolations in tree trunks and submerge vegetation. Konvophoron, Cyanarcus and Pilgeria only were isolate from water samples inoculated in culture media WC and in few opportunities, while three Leptolyngbya species and four Phormidium species were isolated very often. At the stabilization ponds Phormidium sp4 was dominant in 25 of 28 sampling while in the last others were the chlorophycea I. In this study were observed an increase in the frequency of cyanobacteria at the higher contamination places, and a species substitution between different sampling points. There were no biomass studies, therefore is not possible to relate between different cyanobacteria species and some specific types of water quality.
\end{abstract}

Key words: Cyanobacteria, Cyanophyta, pollution, water quality, bioindica ors, waste water, blue-green algae.

Muchas especies de cianobacterias han sido encontradas en aguas eutróficadas y parece ser que dichos organismos son más abundantes en este tipo de aguas que en aguas oligotróficas (Gerloff y Skoog 1957, Branco 1965, Shapiro 1973, Schindler 1974, Fredrickson y Stephanopoulos 1981, Codd et al. 1989, Margalef 1983, Branco 1984, Almeida y Branco 1985, Luttembarck y Rocha 1986, Smith et al. 1987, Stabel 1988, Himberg et al. 1989, Komárek 1991, Stabel 1991, Beyruth 1993, Peinador 1994, Anónimo 1996, González y Ortaz 1998).

Esta mayor abundancia de cianobacterias en aguas eutroficadas puede deberse a uno o varios factores, dentro de los cuales cabe mencionar la capacidad que tienen, las cianobacterias en general, para desarrollarse en ambientes con bajas concentraciones de dióxido de carbono; condiciones que se dan en aguas que poseen altas densidades de algas producto del enriquecimiento de nutrientes (Shapiro 1973, Schindler 1974, Branco 1984, Almeida y Branco 1985, Komárek 1991, Blomqvist et al. 1994); además de poder desarrollarse con concentraciones de dióxido de carbono relativamente bajas, la cubierta musilaginosa de las cianobacterias les ayuda a retenerlo (Margalef 1983). Otro factor que influye para que algunos géneros de cianobacterias, como Microcystis, sean muy numerosas en este tipo de aguas es que, por su modo de vida y 
por los pigmentos fotosintéticos que poseen, aprovechan mejor la luz e impiden el paso de la misma a los estratos inferiores de la masa de agua impidiendo el desarrollo masivo de muchas clorofíceas (Beyruth 1993).

Además de los factores ya mencionados Fredrickson y Stephanopoulos (1981) mencionan la posibilidad de que las sustancias alelopáticas, que segregan muchas cianobacterias, favorezca su desarrollo en este tipo de aguas, al eliminar la competencia de muchos organismos por el medio y al evitar ser depredadas por otros.

Amplias discusiones sobre este tópico se dan en Shapiro (1973), Schindler (1974), Branco (1984), Almeida y Branco (1985), Komárek(1991), y Blomqvist et al. (1994).

\section{MATERIALES Y MÉTODOS}

Seleccioné dos ríos, Cañas y Liberia, que reciben las descargas de sistemas lagunares de estabilización que tratan las aguas residuales domésticas de las ciudades del mismo nombre respectivamente. En cada río escogí tres sitios de muestreo muy cercanos entre sí y que presentaran condiciones de contaminación diferentes.

Realicé 29 muestreos en cada sitio seleccionado de la siguiente manera: tanto para el sistema Cañas como en el sistema Liberia la estación uno la establecí $40 \mathrm{~m}$ aguas arriba de la descarga de las lagunas de estabilización, la estación dos en la descarga de las lagunas y la tres $100 \mathrm{~m}$ aguas abajo de la descarga. Además de estas tres estaciones, seleccioné una cuarta estación en la salida de las lagunas de cada sistema, para poder evaluar las cianobacterias que aportan las lagunas de estabilización a los respectivos ríos.

El río Cañas está situado a $196 \mathrm{~km}$ al norte de la ciudad de San José $\left(10^{\circ} 25^{`}\right.$ N, $85^{\circ} 07^{`}$ W) a 196 msnm y con una temperatura media normal que oscila entre $26.9^{\circ} \mathrm{C}$ y $29{ }^{\circ} \mathrm{C}$ (López et al.1989) y el río Liberia a $235 \mathrm{~km}$ también al norte de la ciudad de San José $\left(10^{\circ} 38^{\circ}\right.$ $\left.\mathrm{N}, 85^{\circ} 27^{\circ} \mathrm{W}\right)$ a $200 \mathrm{msnm}$ y con una temperatura media normal que oscila entre $23^{\circ} \mathrm{C}$ y $32{ }^{\circ} \mathrm{C}$ (López et al. 1989).
En cada estación de muestreo tomé una muestra del agua (A) en botellas de vidrio transparente de $250 \mathrm{ml}$ con tapa esmerilada y las transporté al laboratorio a temperatura ambiente. Una vez en el laboratorio (máximo 24 hr después de tomada la muestra) identifiqué las cianobacterias directamente de la muestra, en los casos que hubo que concentrarlas utilicé mallas de nylon de $2 \mu \mathrm{m}$ a $60 \mu \mathrm{m}$. Para identificar las cianobacterias cuyas poblaciones estaban muy diluidas, o que sólo estaban presentes sus formas reproductoras resistentes, sembré la muestra en medio $\mathrm{F}_{2}$ modificado para agua dulce WC (M) (Guillard 1976). Agregué dos $\mathrm{ml}$ de muestra homogeneizada a $20 \mathrm{ml}$ de medio WC en tubos de ensayo de $22 \mathrm{~mm} \mathrm{X}$ $150 \mathrm{~mm}$ y los dejé en reposo, con luz natural y a temperatura ambiente, hasta obtener crecimiento (aproximadamente 15 días). Para identificar especies de las cuales se necesita conocer los heterocistes las sembré en medio de cultivo BG11 0 (B) (Rippka et al. 1979).

En 19 muestreos coloqué en cada punto tres sustratos artificiales (S) de vidrio transparente de $0.1 \mathrm{~cm}$ por diez $\mathrm{cm}$ por cinco $\mathrm{cm}$ y los dejé a una profundidad de diez $\mathrm{cm}$ dentro del agua por espacio de 15 días a un mes, de modo que en cada muestreo recogía los sustratos colocados en el muestreo anterior. En los ocho últimos muestreos no realicé este paso ya que el tiempo entre un muestreo y otro era muy extenso. Los sustratos recolectados los trasladé al laboratorio dentro de bolsas plásticas sumergidos en agua destilada estéril. El traslado lo realicé a temperatura ambiente y una vez en el laboratorio los coloqué directamente en un microscopio invertido para la identificación de las cianobacterias.

En cada muestreo y punto de muestreo recolecté varias piedras $(\mathrm{P})$, pedazos de troncos o palos y vegetación sumergida o semisumergida (T) y los transporté al laboratorio de la misma forma que los sustratos artificiales. En el laboratorio se raspaban los sustratos, con un escalpelo o cepillo para la identificación de las cianobacterias.

Para la identificación de las cianobacterias utilicé las claves taxonómicas de Geitler (1933), 
Bakes y Bold (1970), Prescott (1970), Rippka et al. (1979), Huber et al. (1983), Komárek (1984), Komárek y Anagnostidis (1986, 1986 A) y Anagnostidis y Komárek $(1988,1990)$.

Todos los organismos identificados se encuentran como organismos testigo en la algateca del Departamento de Biología del Instituto Costarricense de Acueductos y Alcantarillados.

Para la evaluación físico-química y microbiológica de los diferentes puntos de muestreo tomé como referencia los datos presentados en Peinador (1995).

\section{RESULTADOS}

La identificación taxonómica de los organismos la hice hasta género (Cuadros 1 y 2) ya que no fue posible corroborar, con algún especialista, la identificación hasta especie. Cuando encontré más de una especie perteneciente al mismo género procedí a separarlas por monotipos identificándolas por medio de números. La lista sin corroborar de las cianobacterias encontradas las presento en el cuadro cinco.

Aunque el número de especies encontradas es alto, en varios de los sustratos analizados se encontraron muy pocas especies como por ejemplo en troncos y en vegetación sumergida o semisumergida. Konvophoron, Cyanarcus y Pilgeria se localizaron en muy pocas oportunidades y únicamente cuando crecieron en alguno de los medios de cultivo utilizados; otra especie que únicamente se encontró creciendo en medio de cultivo (salvo en una oportunidad) fue Jaaginema sp. 2 (Cuadros 1 y 2).

Como una muestra del perifiton existente en los diferentes lugares tomé como referencia únicamente el número de muestras (frecuencia), con al menos una especie de cianobacteria, recolectadas en piedras, troncos o palos, vegetación sumergida o semisumergida y sustrato artificial (cuadro uno y dos). Hay mayor número de muestras positivas en Cañas río abajo y Cañas descarga que en Cañas río arriba, también en Cañas descarga que en Cañas río abajo y en Liberia descarga que en Liberia río arriba (t-Student, $\mathrm{p}<0.05$ ).

Unicamente hay mayor número de especies en la estación de muestreo Cañas descarga que en Cañas río arriba (Cuadro 3) (t-Student, $\mathrm{p}<0.05$ ). Durante la época seca hay mayor número de muestras positivas en Cañas río abajo y en Cañas descarga que en Cañas río arriba y en Liberia descarga que en Liberia río arriba ( $t-$ Student, $\mathrm{p}<0.05$ ). Así mismo durante la época lluviosa solamente encontré un mayor número de muestras positivas en Cañas río abajo y en Cañas descarga que en Cañas río arriba.

Hay mayor número de especies en Cañas descarga que en Cañas río arriba (U de MannWhiney, $\mathrm{p}<0.05$ ), tanto si se toma en cuenta todos los muestreos o si se toma en cuenta sólo los muestreos realizados en época seca pero no si se toma en cuenta solo los muestreos de época lluviosa.

Microcystis sp.1, Microcystis sp.2 y Oscillatoria sp.2 se aislaron mayormente durante la época seca y Calothrix sp.1, Merismopedia, Microcystis sp.3, Leptolyngbya sp.3, Oscillatoria sp.1, Pseudanabaena sp.1, Phormidium sp.6, Phormidium sp.9, y Planktothrix sp. se aislaron mayormente durante la época lluviosa (Chi, $\mathrm{p}<$ 0.05). Cyanodictyon, Cyanarcus y Konvophoron sólo fueron aisladas en época lluviosa.

Los lugares que muestran un mayor grado de similitud entre sí (Jaccard) son las lagunas de estabilización de ambos sistemas (Cuadro 4).

Los caudales para el río Cañas fueron un máximo de $3940 \mathrm{l} / \mathrm{s}$ y un mínimo de $839 \mathrm{l} / \mathrm{s}$ (Anónimo 1993) y de 3960 l/s el máximo y 301/s el mínimo para el río Liberia (Anónimo 1993).

\section{DISCUSIÓN}

En todos los análisis, salvo que se indique otra cosa, se toma on en cuenta únicamente las cianobacterias que se encontraron fijas a algún tipo de sustrato. Esto obedece al hecho de que se considera que los ríos no tienen plancton propio (Margalef 1983), sino más bien, por influencia de puntos de producción. Al 


\section{CUADRO 1}

Frecuencia total de muestras con cianobacterias en los diferentes tipos de sustratos en el sistema Cañas.

\begin{tabular}{|c|c|c|c|c|c|c|c|c|c|c|c|c|c|c|c|c|c|c|c|c|c|c|c|c|c|c|}
\hline Género & & & & Arrib & & & & & Lagu & una & & & & & & escar & & & & & & & Abajo & & & \\
\hline & $\mathrm{P}$ & $\mathrm{T}$ & $\mathrm{V}$ & A & $\mathbf{M}$ & B & $S$ & $\mathrm{P} \mathrm{V}$ & A & M & B & $\mathrm{S}$ & $\mathrm{P}$ & $\mathrm{T}$ & $\mathrm{V}$ & A & M & B & $\mathrm{S}$ & $\mathrm{P}$ & $\mathrm{T}$ & $\mathrm{V}$ & A & M & B & $S$ \\
\hline Ansbaena 1 & & & & & & & & & & 2 & 3 & & & & & 3 & 4 & 1 & & & & & 2 & 6 & 2 & \\
\hline Ansbaena 2 & & & & & & & & & & & & & & & & & & & & 17 & & & 7 & 20 & 19 & 1 \\
\hline Aphanocapsa & & & & & & & & & 13 & 17 & & & & & & & 3 & & & & & & & & & \\
\hline Calothrix & 2 & & & & 3 & 1 & & & & & & & & & & & 3 & & & 4 & & & & 9 & 2 & \\
\hline Chroococcus & & & & & & & & 5 & 13 & & 1 & & & & & & & & & & & & & & & \\
\hline Gloeocapsa 1 & & & & & & & & & 15 & 18 & & 5 & 1 & & & 9 & 16 & & & & & & 2 & 5 & & \\
\hline Gloeocapsa 2 & & & & & & & & & 16 & 17 & & & & & & 3 & & & & & & & & & & \\
\hline Hapalosiphon & & & & & & & & & & 1 & & 2 & 5 & & 3 & & 6 & 4 & & 2 & & & & & 1 & \\
\hline Jaaginema 1 & & & & & & & & & & 2 & & & & & & & & & & & & & 10 & 17 & & \\
\hline Jaaginema 2 & & & & & & & & & & & & & & & & & & & & & & & & 3 & & \\
\hline Leptolyngbya 1 & & & & & & & & & 7 & 11 & & & 1 & & & 3 & 9 & & & & 2 & & 7 & 8 & & \\
\hline Leptolyngbya 2 & & & & & & & & & 6 & 11 & & & & & & & & & & & & & & & & \\
\hline Leptolyngbya 3 & 3 & & & & 6 & & 1 & & 6 & 15 & & 2 & 4 & & & 11 & 17 & & 3 & & & & 1 & & & \\
\hline Limnothrix & & & & & & & & & 1 & 7 & & & & & & & & & & & & & & & & \\
\hline Lyngbya 1 & & & & & & & & & 1 & 3 & & & 9 & & & 6 & 13 & & 3 & 1 & & & & & & \\
\hline Lyngbya 2 & & & & & & & & & & 5 & & & 2 & & & & 6 & & & & & & & & & \\
\hline Microcystis 1 & & & & & & & & & 3 & 5 & & . & & & & & & & & & & & & & & \\
\hline Microcystis 2 & & & & & & & & & 8 & 16 & & & & & & & & & & & & & & & & \\
\hline Microcystis 3 & & & & & & & & & & & & & & & & & & & & & & & 9 & 12 & & \\
\hline Microcystis 4 & & & & & & & & & & & & & & & & & & & & & & & 2 & 5 & & \\
\hline Nodularia & & & & & & & & & & 2 & & & & & & 1 & 3 & 2 & & & & & & & & \\
\hline Oscillatoria 1 & & & & & & & & & 12 & 16 & & 4 & 2 & I & & 3 & 5 & & & & & & & & & \\
\hline Oscillatoria 2 & 2 & 1 & & 1 & 4 & & & & & & & & 1 & & & & 2 & & & & & & & & & \\
\hline Oscillatoria 3 & 1 & & & & 6 & & & & & & & & & & & & 4 & & & & & & & & & \\
\hline Phormidium 1 & 3 & & & & 5 & & 1 & & & & & & & & & & & & & & & & & & & \\
\hline Phormidium 2 & & & & & & & & & & & & & & & & & & & & & 3 & 1 & 7 & 16 & & \\
\hline Phormidium 3 & & & & & & & & & 7 & 17 & & 9 & & & & 2 & 3 & & & & & & & & & \\
\hline Phormidium 4 & & & & & & & & & 28 & 2 & & 10 & 7 & & & 29 & 1 & & & & & & 5 & 1 & & \\
\hline Phormidium 5 & & & & & & & & & & 1 & & & 3 & & & 12 & 12 & & & 2 & & & 11 & 15 & & 2 \\
\hline Phormidium 6 & & & & & & & & & 1 & 3 & & & 2 & & & 5 & 8 & & & & & & & 3 & & \\
\hline Planktothrl & & & & & & & & & 3 & 8 & & 2 & & & & & & & & & & & & & & \\
\hline Porphyrosiphon & & & & & & & & & 3 & 7 & & 1 & & & & & & & & & & & & & & \\
\hline Pseudanabaena 1 & & & & & & & & & & & & & & & & 8 & 11 & & & & & & 5 & 9 & & \\
\hline Pseudanabaena 2 & & & & & & & & & & & & & & & & & & & & & & & 3 & 6 & & \\
\hline Synechococcus 1 & & & & & & & & & & & & & & & & & 4 & & & & & & 1 & 20 & & \\
\hline Synechococcus 2 & & & & & & & & & & 4 & & & & & & & & & & & & & & & & \\
\hline Synechocystis & & & & & & & & & 14 & 23 & & & & & & & & & & & & & & & & \\
\hline
\end{tabular}




\section{CUADRO 2}

Frecuencia total de muestras con cianobacterias en los diferentes tipos de sustratos en el sistema Liberia.

Género

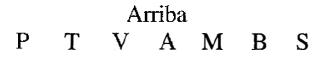
Laguna
V A M B S
Descarga
Abajo
P T V A M B S

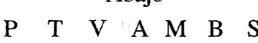

Aphanocapsa 1

Aphanocapsa 2

Aphanothece 1

Aphanothece 2

Calothrix 2

Chroococcus

Cyanaecus

Cyanodictyon

Gloeocapsa 1

Hapalosiphon

Jaaginema 2

Konvophoron

Leptolyngbya 1

Leptolyngbya 2

Leptolyngbya 3

Leptolyngbya 4

Limnothrix

Lyngbya 1

Merismopedia 1

Merismopedia 2

Microcystis 1

Microcystis 2

Microcystis 5

Oscillatoria 2

Phormidium 3

Phomidium 4

Phormidium 5

Phornidium 7

Phormidiun 8

Phormidium 9

Pilgeria

Planktolyngbya

Plankiothrix

Pseudanabciena 1

Pseudanabaena 3

Pseudanabaena 4
$15 \quad 21$

1) 16

$\begin{array}{lll}3 & 5 & 18\end{array}$

$2 \quad 16 \quad 3$

$\begin{array}{ll}7 & 14\end{array}$

310

3

211

24

58

$\begin{array}{lll}6 & 12\end{array}$

36

2

23

$16 \quad 22$

$\begin{array}{ll}7 & 14\end{array}$

67

(13

$\begin{array}{lll}5 & 17 & 3\end{array}$

$17 \quad 21 \quad 5$

$2 \quad 13$

92

21

68

$4 \quad 1$

110

175

616

$11 \quad 17$

58

35

83

31

210

26

14

314

$12 \quad 14 \quad 26 \quad 3$

123

$\begin{array}{llll}5 & 6 & 14 & 3\end{array}$ 
tomar únicamente a las cianobacterias que estaban fijas a algún sustrato (perifiton) se garantiza que esa especie está en ese lugar y persiste en él, porque las condiciones del medio se lo permiten o dicho de otra forma, porque las condiciones del medio se encuentran dentro del ámbito de tolerancia de la especie y esta puede sobrevivir y reproducirse en ese medio.

La razón por la que algunas cianobacterias se aislaron únicamente cuando crecieron en algún medio de cultivo es porque están presentes en concentraciones muy bajas, por lo que es difícil detectarlas directamente del agua, posiblemente el medio les es adverso la mayor parte del tiempo o no pueden competir eficientemente con otros organismos en ese medio.

El hecho de que en el río Cañas la frecuencia de muestras positivas sea significativamente menor en la estación de muestreo río arriba que en las otras dos estaciones corresponde con el mayor grado de contaminación orgánica existente en las estaciones localizadas en la descarga y abajo, producto del efluente de las lagunas de estabilización. Similarmente sucede entre las estaciones de muestreo Cañas descarga y Cañas río abajo.

El caudal del río Cañas disminuye considerablemente en época seca lo cual provoca una menor dilución de los efluentes de las lagunas y por tanto una mayor contaminación en las estaciones de muestreo Cañas descarga y Cañas abajo, esto explicaría el porqué durante esta época hay mayor frecuencia de cianobacterias en esas estaciones de muestreo con respecto a Cañas arriba y no hay diferencia significativa entre la descarga y abajo. Contrariamente el aumento en la dilución de la materia orgánica de los efluentes, provoca una menor contaminación aguas abajo de dicho río, esta menor contaminación pareciera reflejarse en la disminución de la frecuencia de cianobacterias encontrada durante la época lluviosa en esa estación de muestreo.

En el río Liberia la frecuencia de cianobacterias encontradas no refleja, tan claramente como en el río Cañas, la relación que podría existir entre éstas y el grado de contaminación, esto se debe a que en el río Liberia se presenta un grado de contaminación alto en la estación de muestreo colocada aguas arriba (Peinador 1995) por lo cual las cianobacterias ya están presentes en gran número en esa estación.

En cuanto al número de especies de cianobacterias encontradas en las diferentes estaciones de muestreo en ambos ríos, el hecho de que únicamente se observara un aumento en la estación Cañas descarga con respecto a la estación aguas arriba del mismo río y principalmente en época seca, no es suficiente evidencia para aseverar que con similares condiciones físicas (luz, temperatura, velocidad de la corriente etc) se de un aumento en el número de especies de cianobacterias con el aumento de la contaminación orgánica.

Sin embargo a pesar de que estadísticamente, no se refleje un aumento en el número global de especies de cianobacterias conforme aumenta la contaminación en las diferentes estaciones de muestreo colocadas en el río Cañas, analizando los datos obtenidos en cada muestreo individualmente, encontré que el número de especies aisladas siempre fue mayor en la estación aguas abajo que en la estación aguas arriba, por lo tanto, si tomo como variable el número de muestreos en que se encontraron mayor número de especies de cianobacterias en lugar de, el número global de especies encontradas en cada lugar, obtengo que si se verifica un aumento en el número de especies de cianobacterias en lugares con mayor contaminación.

Entre las estaciones de muestreo descarga y aguas abajo, siempre en el río Cañas, si lo analizamos también de esta última manera no obtenemos diferencia significativa. Esto puede deberse a que existe una mayor similitud, en cuanto a grado de contaminación, entre estos dos lugares que entre abajo y arriba (Peinador 1995).

Analizando la similitud de lugares por medio del índice de Jaccard, tomando en cuenta solo las especies del perifiton, excepto para las lagunas en las cuales se toman en cuenta también las especies encontradas en el agua, por ser sistemas de aguas prácticamente estancadas, notamos que tanto entre las estaciones de muestreo aguas arriba y aguas abajo del río Liberia 


\section{CUADRO 3}

Resultado de los muestreos según época seca y lluviosa con especies fijas a algún sustrato.

$$
\text { Arriba }
$$

Cañas

Descarga

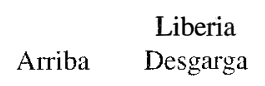

Abajo

\begin{abstract}
Muestreos época lluviosa
Muestreos época seca
Muestras tomadas en época lluviosa
Muestras tomadas en época seca
Muestras positivas en época lluviosa
Muestras positivas en época seca
Especies en época lluviosa
Especies en época seca
Número total de especies
uestreos época lluviosa

Muestras tomadas en época lluviosa

Muestras positivas en época lluviosa

Número total de especies
\end{abstract}

$\begin{array}{rrrrrr}17 & 17 & 17 & 17 & 17 & 17 \\ 12 & 12 & 12 & 12 & 12 & 12 \\ 61 & 61 & 61 & 61 & 61 & 61 \\ 45 & 45 & 45 & 45 & 45 & 45 \\ 11 & 29 & 16 & 17 & 26 & 23 \\ 3 & 17 & 14 & 1 & 20 & 15 \\ 5 & 11 & 5 & 4 & 7 & 6 \\ 1 & 8 & 4 & 3 & 7 & 7 \\ 5 & 11 & 7 & 4 & 8 & 9\end{array}$

\section{CUADRO 4}

Indice de Jaccard con especies fijas a algín sustrato.

$\begin{array}{lrrrrrrr} & \text { Laguna } & \begin{array}{c}\text { Cañas } \\ \text { Arriba }\end{array} & \text { Abajo } & \text { Descarga } & \text { Laguna } & \begin{array}{r}\text { Liberia } \\ \text { Arriba }\end{array} & \text { Abajo } \\ \text { DESCARGA } & 0.5 & 0.24 & 0.42 & 0.21 & 0.32 & 0.25 & 0.29 \\ \text { LAGUNA } & - & 0.08 & 0.23 & 0.31 & 0.58 & 0.00 & 0.29 \\ \text { ARRIBA } & - & - & 0.16 & 0.16 & 0.00 & 0.22 & 0.00 \\ \text { ABAJO } & - & - & - & 0.14 & 0.23 & 0.18 & 0.38 \\ \text { LIBERIA } & - & - & - & - & 0.38 & 0.36 & 0.5 \\ \text { DESCARGA } & - & - & - & - & - & 0.17 & 0.36 \\ \text { LAGUNA } & - & - & - & - & - & - & 0.16 \\ \text { ARRIBA } & - & - & - & - & & & \end{array}$

En las lagunas se toman en cuenta también las especies encontradas en el agua

como entre esas mismas estaciones en el río Cañas hay un índice de similitud de 0.16 , esto nos indica que en ambos ríos ocurre un cambio muy notorio entre esos dos puntos por influencia de las descargas de las lagunas de estabilización.

Conjuntamente con esa disimilitud observada entre esos sitios de muestreo, el incremento del índice de similitud entre la descarga y aguas abajo en ambos ríos, reafirma la influencia de los efluentes de las lagunas sobre los ecosistemas respectivos y si bien es cierto que, en términos generales, el aumento en el número de especies de cianobacterias no es significativo entre un lugar y otro, si se evidencia una sustitución de especies.
Aunque para evaluar la calidad del agua en un río es mejor tomar en cuenta sólo las especies del perifiton, en términos de efectividad de depuración y funcionamiento de los ecosistemas operantes, es válido tomar en cuenta a todas las especies presentes pues de una $\mathrm{u}$ otra forma afectan el ecosistema. $\mathrm{Si}$ tomamos en cuenta todas las especies de cianobacterias, este cambio, entre aguas arriba y aguas abajo en ambos ríos, determinado mediante el análisis de los datos y causado por las descargas de las lagunas, es más notorio.

Cambios como los observados en el número de especies, frecuencia de muestras positivas y/o sustitución de especies implican 
necesariamente un cambio en la comunidad general de organismos ya que no es prudente esperar que sólo la comunidad de cianobacterias cambie su estructura como respuesta a las condiciones del medio.

La proliferación de cianobacterias, por si mismas, también puede tener un profundo efecto sobre la estructura comunitaria de los organismos en general. Ellas, entre otros efectos, inhiben por medio de toxinas, el crecimiento de algas que sirven de alimento a muchos organismos (Gonzáles 1988). Se han identificado varios de esos inhibidores como muscarinas y en el caso de Nostoc, dihidrooxiantraquinona (Margalef 1983). Gonzáles (1988) cita otros efectos que produce el aumento de cianobacterias en un ecosistema acuático.

Retomando los resultados obtenidos mediante el índice de Jaccard observamos que los lugares con mayor semejanza entre sí son las lagunas, esto es lo esperado dado que son sistemas artificiales muy semejantes físicamente, operando en condiciones meteorológicas similares y con afluentes de la misma naturaleza química; su disimilitud se basa en condiciones de suelo, manejo y carga afluente. En cuanto a la similitud entre los diferentes sitios de muestreo de los ríos, prevalecen los valores bajos y en términos generales, se puede decir que los diferentes puntos de muestreo son muy diferentes entre sí.

Sin embargo hay una tendencia de aumento de similitud si comparamos los sitios de muestreo de los dos ríos de la siguiente manera: aguas arriba de ambos ríos tienen un valor de cero, las descargas $0.21 \mathrm{y}$ aguas abajo 0.38 , este hecho puede obedecer a diferentes factores o combinación de ellos. Las especies que van permaneciendo son las más tolerantes a las condiciones del medio, por lo que poseen mayor resistencia a la sedimentación y difusión y/o las especies que van permaneciendo son las más frecuentes en el o los sitios de muestreo anteriores (menor dilución). De las especies que están presentes aguas abajo de ambos ríos, diez son las más frecuentes en las descargas, de esas diez, ocho están en común en ambos ríos y el resto sólo aparece aguas abajo de uno u otro río, además esas ocho especies en común son las más frecuentes en ambos ríos; por lo anterior se puede deducir que las especies que van permaneciendo son las más abundantes en los sitios de muestreo anteriores, sin descartar otras razones.

Otros de los sitios en que cabría esperar una mayor similitud es en el caso de las descargas y las lagunas en ambos ríos sin embargo, aunque si se nota un incremento en los valores del índice con respecto a los valores entre otros puntos de muestreo, el cambio en las condiciones generales del medio entre la laguna y el río es grande (dilución, sedimentación, velocidad de la corriente, etc.) y esto influye en buena parte para que esto no se dé.

En términos generales y tomando en cuenta todas las cianobacterias, encontré mayor número de especies en la época lluviosa. Solamente tres especies fueron más abundantes en época seca. Las razones para este hecho podrían ser muchas ya que están involucrados aspectos de fisiología, morfología, sucesiones y ritmos (Margalef 1983).

Durante la realización de este trabajo, en las lagunas de estabilización tanto de Cañas como de Liberia, siempre fue muy notoria la dominancia de la cianobacteria Phormidium sp.4, salvo en tres ocasiones en las cuales, y en ambos sistemas a la vez, dominó la clorofícea Chlorella vulgaris, por el hecho de que esta dominancia se dio en ambos sistemas a la vez, no puede obedecer a condiciones propias de cada sistema, sino más bien, a factores climáticos (dada la cercanía de los lugares) o a factores intrínsecos de la especie ya que he observado ese mismo comportamiento en otras especies cultivadas bajo condiciones de laboratorio.

No es posible hacer comparaciones entre las cianobacterias encontradas en los diferentes sitios de muestreo, con las listas que aparecen en la literatura y que definen las especies y las asociaciones más frecuentemente encontradas en las diferentes zonas de contaminación ya 


\section{CUADRO 5}

Lista tentativa de especies de cianobacterias

\begin{tabular}{|c|c|}
\hline a flos & \\
\hline Anabaenasp. & (Anabaena 2) \\
\hline \multicolumn{2}{|l|}{ Aphanocapsa elachista var. } \\
\hline Planctonica & (Aphanocapsa 1$)$ \\
\hline Aphanocapsa delicatissima & (Aphanocapsa 2) \\
\hline Aphanothece clathrata & (Aphanothec \\
\hline Aphanothece nidulans & (Aphanothece 2) \\
\hline Calothrix parietina & Caloth \\
\hline Calothrix sp. & (Calothrix 2) \\
\hline cus minu & coccus $\mathrm{sp}$. \\
\hline yar & $\lfloor$ Cyc \\
\hline latus & \\
\hline loe & psa 1$)$ \\
\hline Gloec & psa 2) \\
\hline apc & iphon $\mathrm{s}$ \\
\hline ag & (Jaa \\
\hline Jaaginema subtilissimun & $1 \mathrm{Jaa}$ \\
\hline \multicolumn{2}{|l|}{ Konvophoron sp. (sbg. } \\
\hline Alyssophoron) & oron $\mathrm{sp}$ ) \\
\hline Leptoly & olyngbya 1 ) \\
\hline epto & gbya 2) \\
\hline Lepto & (Leptolyngbya 3$)$ \\
\hline Leptol & (Leptolyngbya 4$)$ \\
\hline Limnothrix sp. & (Limnothrix sp.) \\
\hline Lyngbya martensiana & (Lyngbya 1$)$ \\
\hline Lyngbya sp. & (Lyngbya 2$)$ \\
\hline Merismopedi & pedia 1 ) \\
\hline the & $(\mathrm{Mer}$ \\
\hline flos-aql & $\mathrm{MiC}$ \\
\hline stis margi & (Microcystis 2) \\
\hline
\end{tabular}

que, estas listas son incompletas pues al igual que las encontradas en este estudio, son confeccionadas para lugares específicos y están lejos de ser universales. Así mismo, una especie puede encontrarse en diferentes condiciones de contaminación dependiendo de otras características del medio o pueden ser variedades diferentes de la misma especie. Esto último es muy probable dado el desconocimiento taxonómico que existe sobre este grupo de organismos en el trópico, sin embargo y en términos generales, la mayoría de los géneros de cianobacterias encontradas en este estudio, han sido reportadas por uno o más autores como típicas de aguas contaminadas con materia orgánica.

Microcystis pulverea
Microcystis sp. 1
Microcystis sp. 2
Nodularia harveyana
Oscillatoria chalybea
Oscillatoria sp. 1
Oscillatoria sp. 2
Phormidium aerugineo-coeruleum
Phormidium amphibium
Phormidium diguetii
Phormidium irriguum
Phormidium retzii
Phormidium tinctorium
Phormidium favosum
Phormidium formosum
Phormidium numidicum
Pilgeria brasiliensis
Planktolyngbya subtilis
Planktothrix rubescens
Porphyrosiphon versicolor
Pseudanabaena catenata
Pseudanabaena sp. (sbg. Ilionema)
Pseudanabaena sp. 3
Psedanabaena sp. 4
Synechococcus cedronum
Synechococcus sp.
Synechocystis sp.

(Microcystis 3)

(Microcystis 4)

(Microcystis 5)

(Nodularia $\mathrm{sp}$ )

(Oscillatoria 1)

(Oscillatoria 2)

(Oscillatoria 3)

(Phormidium 1)

(Phormidium 2)

(Phormidium 3)

(Phormidium 4)

(Phormidium 5)

(Phormidium 6)

(Phormidium 7)

(Phormidium 8)

(Phormidium 9)

(Pilgeria sp.)

(Planktolyngbya sp.)

(Planktothrix sp.)

(Porphyrosiphon sp.)

(Pseudanabaena 1)

(Pseudanabaena 2)

(Pseudanabaena 3)

(Pseudanabaena 4)

(Synechococcus 1)

(Synechococcus 2)

(Synechocystis sp.)

\section{AGRADECIMIENTOS}

Agradezco al Instituto Costarricense de Acueductos y Alcantarillados su apoyo logístico.

\section{RESUMEN}

En dos ríos de Costa Rica que sirven como cuerpos receptores de aguas residuales domésticas, tratadas mediante lagunas de estabilización, se realizó un total de 28 muestreos en tres puntos de cada río y en las lagunas por un período de cinco años y medio. En cada nuestreo se recolectaron muestras de cinco tipos de sustratos y se cultivó una en dos tipos de medios artificiales. Se observó entre otras cosas, un aumento en la frecuencia de cianobacterias en los lugares con mayor contaminación y una sustitución de especies entre los diferentes lugares. 


\section{REFERENCIAS}

Almeida, A. R. \& S. M. Branco. 1985. O nitrogênio e fósforo como fatores limitantes ao desenvolvimento de algas cianofíceas na represa Billings. Dae 141:156-158.

Anagnostidis, K. \& J. Komárek. 1990. Modern approach to the classification system of cyanophytes (Stigonematales). Arch. Hydrobiol. Suppl. 59:1-73.

Anagnostidis, K. \& J. Komárek. 1988. Modern approach to the classification system of cyanophytes Oscillatoriales. Arch. Hydrobiol. Suppl. 5053:327-472.

Anónimo. 1976. Investigación de parámetros en las lagunas de estabilización de Cañas y Liberia. Instituto Costarricense de Acueductos y Alcantarillados, San José, Costa Rica. 29p.

Anónimo. 1991. Caudales de cuencas hidrográficas. Instituto Costarricense de Electricidad. Boletín Hidrológico, Costa Rica. 19: 214.

Anónimo. 1996. Guidelines for drinking water quality, Vol. 2. WHO, Ginebra. p. 75-77.

Bakes, A .F. \& H. C. Bold. 1970. Phycological studies. X taxonomics studies in the oscillatoriaceae. University of Texas, Austin. 104 p.

Beyruth, Z. 1993. Fitoplancton em lagos do Parque Ecológico do Tiete, Sao Paulo, 1986-1987. Estudo para reabilitaçao. Dae 170:5-10

Blomqvist, D. O., A. Pettersson \& F. Hyenstrand. 1994. Ammonium-nitrogen: a key regulatory factor causing dominance of non-nitrogen fixing Cyanobacteria in aquatic system. Arch. Hydrobiol. 132: 141164.

Branco, S. M. 1984. Limnología sanitaria, estudio de la polución de aguas continentales. OEA, Washington, D. C. 120 p.

Branco, S. M. 1965. Poluiçáo e contaminaçao. Dae 26:41-45.

Cood, G. A., G. S. Bell \& W. P. Brooks. 1989. Cyanobacterial toxin in water. Wat. Sci. Tech. 21:1-13.

Fredrickson, A .G. \& Stephanopoulos. 1981. Microbial competition. Science 213:972-979.

Geitler, L. 1933. Cyanophyceae. In Rabenhorsts Kryptogamenflora. Akadem. Verlagsgeselich, Leipzig, Alemania 14:1-1196.
Gerloff, G. C. \& F. Skoog. 1957. Nitrogen as limiting factor for the growth of Microcystis aeruginosa in southern Wisconsin lakes. Ecology 38:556-561.

Gonzáles, A. 1988. El fitoplancton de las aguas continentales. OEA, Washington, D. C. 125 p.

González, E. J. \& M. Ortaz. 1998. Efectos del enriquecimiento con $\mathrm{N}$ y P sobre la comunidad del fitoplancton en microcosmos de un embalse tropical (La Mariposa, Venezuela).Rev. Biol. Trop. 46: 27-34.

Guillard, R .R. L. 1976. Culture of phytoplankton for feeding marine invertebrates. Woods Hole Oceanografic Insthetion, Massachusetts. $60 \mathrm{p}$.

Himberg, A. M., A. M. Keijola, L. Husvirta, H. Pyysalo \& K. Sivonem. 1989. The effect of water treatment processes from Microcystis and Oscillatoria cyanobacteria: A laboratory study. Hgt. Rev. 60:1-5

Huber, G., J. Komárek \& B. Fott. 1983. Das phytoplankton des SüBwassers. E. Schweizerbart sche Verlagsbuchhandlung, Stuttgart. $445 \mathrm{p}$.

Komárek, J. 1984. Sobre las cianofíceas de Cuba: Aphanizomenon volzii; (2) especies de Forticea. Acta Botánica Cubana $18: 1-29$.

Komárek, J.1991. A review of water bloom forming Microcystis specvies, with regard to populations from Japan. Algol Stud. 64:115-127.

Komárek, J. \& K. Anagnostidis. 1986. Modern approach to the classification system of cyanophytes (Croococcales). Arch. Hydrobiol. Suppl. 43: 157-226.

Komárek, J. \& K. Anagnostidis. 1986a. Modern approach to the classification system of cyanophytes (Nostocales). Arch. Hydrobiol. Suppl. 56: 247-345.

López, M. M., M. Ruiz y H. Farrer. 1989. Lagunas de estabilización. Instituto Costarricense de Acueductos y Alcantarillados, San José, Costa Rica 60p.

Luttembarck, B. H. B. \& A. A. Rucha. 1986. Autodepuraçao nos cursos da água. Dae 144:27-32.

Margalef, R. 1983. Limnología. Omega, Barcelona. 1010 p.

Peinador, M. 1994. Cianobacterias potencialmente tóxicas en plantas de tratamiento de agua en Costa Rica. Rev. Biol. Trop. 42: 5-8.

Peinador, M. 1995. Potencial impacto del turismo masivo sobre el tratamiento de aguas residuales'en las ciudades de Cañas y Liberia, Guanacaste. Tesis de Licenciatura, Universidad de Costa Rica, San José. 
Prescott, G. W. 1970. The freshwater algae. W.M.C. Brown Company Publishers, Washington, D. C. 282 p.

Rippka, R., J. Deruelles, J. Waterbury, M. Herdman \& R. Stainer. 1979. Generic assignments, strain histories properties of pure cultures of cianobacteria. J. Gen. Microbiol. 111:1-61.

Schindler, D. W. 1974. Eutrophication and recovery in experimental lakes: implications for lakes management. Rev. Science 184:897-988.

Shapiro, J. 1973. Blue-green algae: Why they become dominant. Science 179:382-384.
Smith, V. H., E. Willin \& B. Karlsson. 1987. Predicting the summer peak biomass of four species of blue-green algae (Cyanophyta / Cyanobacterial) in Swedish lakes. Wat. Res. Bull. 23:397-402.

Stabel, H. H. 1988. Algal control of elemental sedimentary fluxes in lake Constance. Verh. Internat. Verein. Limnol. 23:700-706.

Stabel, H. H. 1991. Irregular biomass response in recovering prealpine lakes. Verh. Internat. Verein. Limnol. 24:810-825. 